\title{
Bit-Error Rates for Optical Receivers Using Avalanche Photodiodes with Dead Space
}

\author{
Majeed M. Hayat, Member, IEEE, Bahaa E. A. Saleh, Fellow, IEEE, and John A. Gubner, Member, IEEE
}

\begin{abstract}
Bit-error rates are computed for an on-off keying optical communication system using avalanche photodiodes (APD's). We use a model for the APD that includes dead space and the finite response time. Dead space is the minimum distance that a newly generated carrier must travel in order to acquire sufficient energy to become capable of causing an impact ionization in the multiplication region of the APD. The detector's finite impulse response and its randomness are important for high datarate systems. Using an exact analysis, we show that the presence of dead space enhances the performance at relatively low data rates. Using a Gaussian approximation technique with the exact mean and variance, we demonstrate that dead space degrades the performance at high data rates since it is responsible for longer tails in the impulse response function of the APD, which in turn increases the effect of intersymbol interference.
\end{abstract}

\section{INTRODUCTION}

$\mathbf{N}$ UMEROUS studies have addressed the problem of evaluating the performance of optical communication systems including effects such as quantum noise, noise due to gain fluctuations associated with avalanche photodiodes (APD's), and receiver thermal noise [1]. The effects of dispersion in the optical fiber on pulse broadening and its contribution to intersymbol interference (ISI) were also included in calculating error rates. This paper addresses the performance of on-off keying (OOK) optical communication systems taking into consideration two new effects. First, a more accurate model for the APD's gain-fluctuation noise is used in which dead space is included in the avalanche multiplication process. Second, the effect of the APD's finite (noninstantaneous) response on ISI is also studied. The latter effect becomes important when the data rate is so high that the interbit time interval is comparable to the response time of the detector. Such high data rates are becoming possible as a result of advances in the design of the fibers themselves, by which dispersion is dramatically reduced through the use of linear and nonlinear effects.

A fundamental assumption implicit in most models of APD's is that the impact ionization probability of a carrier is the same at all times, including the instant following its generation, so that this probability is independent of the carrier's history. From a physical point of view, however, a newly generated carrier must travel some distance in order to build up sufficient kinetic energy to enable it to initiate an ionization.

Paper approved by C. N. Georghiades, the Editor for Synchronization and Optical Detection of the IEEE Communications Society. Manuscript received January 14, 1993; revised June 14, 1993. This work was supported by the National Science Foundation.

The authors are with the Department of Electrical and Computer Engineering, University of Wisconsin, Madison, WI USA 53706

IEEE Log Number 9406237
To accommodate this requirement, an avalanche multiplication model has been formulated for which the ionization probability of a carrier is set to zero for a certain distance, called the dead space, immediately following its generation. The actual value of dead space depends on the electric field profile in the multiplication region and the carrier ionization threshold energy.

In an earlier work [2], it was shown that each absorbed photon by the APD induced a random response whose mean had an exponentially decaying tail. Thus, photons in a single optical pulse (representing a bit of the OOK system) generate a stream of random impulse responses. These overlap to produce the photoelectric current corresponding to the optical pulse. This response has a random shape, random duration, and a random area - since both the gain of the APD and the duration of its impulse response function are random. The process of extracting information from the output of the detector is complicated by the randomness of the response to individual optical pulses as well as the random spread of the response to adjacent pulses.

The process is further complicated by the presence of circuit noise from the preamplifier stage which can be modeled as an additive zero-mean Gaussian noise. This noisy output comprises the observed signal based on which the decision as to whether the transmitted bit is "zero" or "one" is made. Our goal is to determine the bit-error rate (BER) of the communication system.

This problem has long been considered by many researchers in the past two decades or so using various approaches. All the previous work dealt with a conventional multiplication model for APD's which neglects dead space. These studies include "exact" calculations [3]-[9], Gaussian approximations [10], [11], Monte Carlo simulations [12], Chernoff bounds [10], and large deviation techniques applied to Monte Carlo simulations [13]. Furthermore, due to the unavailability of analytical theory for the APD's impulse response function, these studies primarily assumed an instantaneous response APD for which the transient tail of the impulse response function of the APD is ignored. This is clearly a reasonable assumption when the transmission rate is much less than the bandwidth of the APD. However, with the recent advances in fiber-optic technology, the effect of channel dispersion is minimized and very high data rates became possible. Our analysis, therefore, focuses on the limitations brought about by the speed of the detector. The detector speed can then play a role in limiting the error rates.

Our contribution in this paper is twofold. We carry out the BER calculations taking into account, for the first time, 

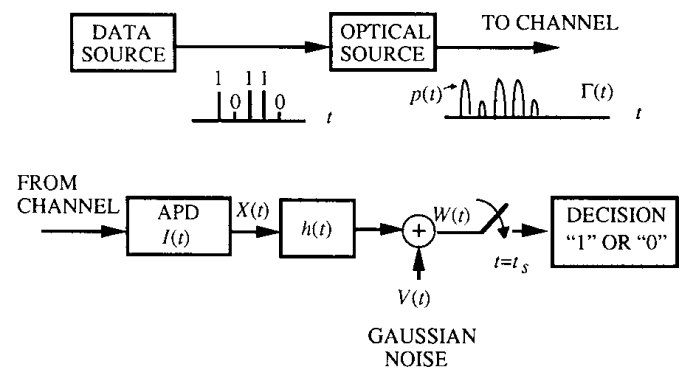

Fig. 1. Block diagram of an on-off keying optical communication system.

the intersymbol interference effect due to the finite width of the APD's impulse response function. We also demonstrate, for the first time, how dead space affects the BER. These calculations are possible now that the statistics of the APD's impulse response function and gain have been determined (see [2], [14], and [15]). Quantum noise and circuit noise are also considered in the analysis. The possible beneficial effect of dead space is then demonstrated through numerical examples.

\section{StATISTICS OF THE RECEIVER OUTPUT}

\section{A. The Optical Signal}

The transmitted optical signal is composed of a modulated stream of identical optical pulses, each of an arbitrarily prescribed deterministic shape $p(t)$, separated by time $T$. The pulse $p(t)$ is assumed to vanish outside the interval $[0, \tau]$ for some $\tau \leq T$, so that the duty cycle of the transmitted message is $\tau / T$. Each of these pulses is multiplied by a random amplitude belonging to a finite alphabet set. The intensity of the transmitted signal is thus given by

$$
\Gamma(t)=\sum_{n=0}^{\infty} B_{n} p(t+n T)
$$

where $\left\{B_{i}\right\}$ is an independent and identically distributed collection of random variables taking values in an alphabet $A$ (e.g., $A=\{0,1\}$ for on-off keying). Each element $q$ in $A$ is chosen with probability $P_{q}=P\left\{B_{n}=q\right\}$ satisfying $\sum_{q \in A} P_{q}=1$. Fig. 1 is a schematic diagram of the OOK communication system which is discussed in this section.

\section{B. The Photoelectric Signal}

The process of photodetection results in the generation of a stream of photoelectrons at times $\left\{\tau_{i}\right\}$ (with reference to $t=0$ ) described by a nonhomogeneous Poisson point process with a rate $\Gamma(t)$. This random nature of photon arrivals constitutes the quantum noise. If a photon arriving at time $\tau_{i}$ generates in the receiver circuit a random photoelectric pulse $I_{i}\left(t-\tau_{i}\right)$, then the total photoelectric current is

$$
X(t)=\sum_{\tau_{i} \leq t} I_{i}\left(t-\tau_{i}\right)
$$

(Each of the processes $I_{i}(t)$ is a random impulse response function of the APD.) Because $\Gamma(t)$ is a stochastic process (governed by the random sequence $\left\{B_{i}\right\}$ which is independent of $\left.\left\{\tau_{i}\right\}\right),\left\{\tau_{i}\right\}$ are realizations of a doubly stochastic Poisson process (i.e., a Poisson process with a random rate). $\left\{I_{i}(\cdot)\right\}$ are assumed to be independent and identically distributed stochastic processes, each of which is assumed to be integrable and statistically independent of the sequence $\left\{B_{i}\right\}$ and the arrival times $\left\{\tau_{i}\right\}$. The process $X(t)$ is a shot noise with a random filter $I(t)$ and generated by anderlying doubly stochastic Poisson point process with random intensity $\Gamma(t)$. (Since the collection $I_{i}(\cdot)$ is identically distributed, we will omit the subscript whenever convenient.)

A simple way to determine the statistics of $X(t)$ is to determine its characteristic function. Conditioned on a fixed sample path of the intensity $\Gamma(t)$, i.e., a fixed sequence $B_{n}=$ $b_{n}, n=0,1,2, \cdots$, the conditional characteristic function $\varphi_{X(t) \mid\left\{B_{n}\right\}}(u)=E\left[e^{j u X(t)} \mid\left\{B_{n}\right\}\right]$ is [16]

$$
\begin{aligned}
\varphi_{X(t) \mid\left\{B_{n}\right\}}(u)=\exp \left\{\int_{-\infty}^{t} \sum_{n=0}^{\infty} B_{n} p(s+n T)\right. \\
\left.\cdot\left[\varphi_{I(t-s)}(u)-1\right] d s\right\} .
\end{aligned}
$$

Thus, the characteristic function of $X(t)$ is simply

$$
\begin{aligned}
\varphi_{X(t)}(u) & =E\left[\varphi_{X(t) \mid\left\{B_{n}\right\}}(u)\right] \\
& =E\left[\exp \left\{\sum_{n=0}^{\infty} B_{n} \phi_{n}(t, u)\right\}\right] \\
& =\prod_{n=0}^{\infty} E\left[\exp \left\{B_{n} \phi_{n}(t, u)\right\}\right] \\
& =\prod_{n=0}^{\infty}\left(\sum_{q \in A} P_{q} \exp \left\{a \phi_{n}(t, u)\right\}\right),
\end{aligned}
$$

where

$$
\phi_{n}(t, u)=\int_{-n T}^{\min \{\tau-n T, t\}} p(s+n T)\left\{\varphi_{I(t-s)}(u)-1\right\} d s .
$$

The characteristic function of $X(t)$ is therefore explicitly related to the alphabet probabilities $P_{q}$ and the characteristic function of the process $I(t)$. The latter is governed by the statistics of the APD's impulse response function, of which a brief discussion is given next.

\section{The APD Impulse Response Function}

Consider a double-carrier-multiplication APD with a certain multiplication-region width with a uniform electric field. Starting at one edge of the multiplication region at time $t=0$, an original electron initiates the avalanche multiplication process. This process is assumed to obey a dead-space multiplication model (see [14]). As a result of the moving offspring (electrons and holes) within the multiplication region, an induced electric current is generated. This time-dependent current, which we denote by $I(t)$, is the random impulse response function of the APD. When all the electrons and holes leave the multiplication region, $I(t)$ vanishes. 
The statistics of $I(t)$, including its mean, standard deviation, and characteristic function (all as functions of time), were determined in [2] using renewal theory techniques. The asymptotic behavior of the mean and the standard deviation for large $t$, which proves to be particularly useful for the BER calculations in Section III, was also determined.

\section{The Receiver Output}

The photoelectric current $X(t)$ acts as a current source in the receiver circuit. If the receiver circuit is modeled by a linear time-invariant filter of impulse response function $h(t)$, and if it contains an additional source of zero-mean Gaussian noise $V(t)$ (e.g., due to thermal noise from resistors), then the final signal on which the decision is based is

$$
\begin{aligned}
W(t) & =V(t)+h(t) * X(t) \\
& =V(t)+\sum_{\tau_{i} \leq t} I_{i}\left(t-\tau_{i}\right) * h(t) \\
& =V(t)+\sum_{\tau_{i} \leq t} \tilde{H}_{i}\left(t-\tau_{i}\right) .
\end{aligned}
$$

Here, $\tilde{H}_{i}$ denotes the response of the filter $h$ when its input is $I_{i}(t)$, the APD's impulse response function due to the $i$ th photon, and the symbol $*$ denotes convolution.

Let $W$ denote the value of $W(t)$ at a certain sampling instant $t=t_{s}$ with $0 \leq t_{s} \leq T$ ( $T$ being the bit length) and let $V \equiv V\left(t_{s}\right)$, then

$$
W=\sum_{\tau_{i} \leq t} \hat{H}_{i}\left(\tau_{i}\right)+V
$$

where

$$
\hat{H}_{i}(t) \equiv \tilde{H}_{i}\left(t_{s}-t\right)
$$

From here on, we restrict our attention to an on-off keying (OOK) modulation scheme with $P\left\{B_{n}=0\right\}=P\left\{B_{n}=\right.$ $1\}=0.5, n=0,1,2, \cdots$. Now by replacing $I_{i}$ by $\tilde{H}_{i}$ in (2)-(4) and by noting the independence of the Gaussian noise, the characteristic function of $W$ is

$$
\varphi_{W}(u)=\varphi_{V}(u) \prod_{n=0}^{\infty}\left(\frac{1}{2}\left\{1+\exp \left[\phi_{n}(u)\right]\right\}\right),
$$

where

$$
\phi_{n}(u)=\int_{-n T}^{\min \left\{\tau-n T, t_{s}\right\}} p(s+n T)\left\{\varphi_{\hat{H}_{i}(t)}(u)-1\right\} d t
$$

and $\varphi_{\hat{H}_{i}(t)}(u)=E\left[e^{j u \hat{H}_{i}(t)}\right]$ is the characteristic function of the random variable $\hat{H}_{i}(t)$.

\section{E. Hypothesis Testing}

Since the system is stationary, there is no loss of generality in determining the probability of error for an arbitrary bit, say when $n=0$. Let $H_{0}$ and $H_{1}$ denote the null and the alternative hypotheses, respectively. Under $H_{0}, B_{0}=0$, and the optical signal is given by $\Gamma(t)=\sum_{n=1}^{\infty} B_{n} p(t+n T)$, while under $H_{1}$, $B_{0}=1$, and $\Gamma(t)=p(t)+\sum_{n=1}^{\infty} B_{n} p(t+n T),-\infty<t \leq T$.
Under $H_{0}$, the characteristic function of $W$ is

$$
\begin{aligned}
\varphi_{W}^{0}(u) & =\varphi_{V}(u) E\left[\exp \left\{\sum_{n=1}^{\infty} B_{n} \phi_{n}(u)\right\}\right] \\
& =\varphi_{V}(u) \prod_{n=1}^{\infty}\left(\frac{1}{2}\left\{1+\exp \left[\phi_{n}(u)\right]\right\}\right),
\end{aligned}
$$

and under $H_{1}$, it is

$$
\begin{aligned}
\varphi_{W}^{1}(u) & =\varphi_{V}(u) E\left[\exp \left\{\phi_{0}(u)+\sum_{n=1}^{\infty} B_{n} \phi_{n}(u)\right\}\right] \\
& =\varphi_{V}(u) \exp \left[\phi_{0}(u)\right] \prod_{n=1}^{\infty}\left(\frac{1}{2}\left\{1+\exp \left[\phi_{n}(u)\right]\right\}\right)
\end{aligned}
$$

where $\varphi_{V}(u)=E\left[e^{j u V}\right]$ is the characteristic function of $V$, and it has the well-known Gaussian form $\varphi_{V}(u)=$ $\exp \left[-\frac{1}{2} \sigma^{2} u^{2}\right]$, where $\sigma^{2}$ is the variance of $V$.

Let $P_{0}(x)$ and $P_{1}(x)$ denote the conditional probability distribution functions of $W$ under the hypotheses $H_{0}$ and $H_{1}$, respectively, i.e., $P_{i}(x)=P\left\{W \leq x \mid H_{i}\right\}, i=0,1$. The corresponding conditional probability density functions of $W$ can be computed by carrying out the Fourier integral

$$
f_{i}(x)=\frac{1}{2 \pi} \int_{-\infty}^{\infty} \varphi_{W}^{i}(u) \exp [-j u x] d x, \quad i=0,1 .
$$

Let us form the likelihood function $L(x)=f_{1}(x) / f_{0}(x)$. The optimum decision rule is thus

$$
L(x) \underset{H_{0}}{\stackrel{H_{1}}{\gtrless}} 1 .
$$

This rule results in two disjoint subsets of the real line. Let the set $D$ be defined so that $L(x) \geq 1, \forall x \in D$, and let $D^{C}$ be its complement. The average BER, defined as the probability of making an error in detection per bit averaged over all possible bit levels is

$$
\text { BER }=\frac{1}{2}\left\{\int_{D} f_{0}(x) d x+\int_{D^{C}} f_{1}(x) d x\right\} .
$$

If $\varphi_{W}^{0}(u)$ and $\varphi_{W}^{1}(u)$ were known, then one might use the techniques in [17] to compute the corresponding density functions and the probability of error efficiently. However, even if the characteristic function of the impulse response $I(t)$ is known, the characteristic function of $\hat{H}_{i}(t)$ is not, in general, analytically computable. Exact evaluation of the BER is, therefore, in general not possible. We will adopt two approximations which will make the calculations possible.

\section{Gaussian Approximation}

This is a simple approximation in which the distribution function of $W$ is approximated by a Gaussian distribution with the exact mean and variance. The validity of this approximation has been verified for high light intensity levels [18]-a good discussion on this subject can be found in [10] and [19]. In our case, the exact mean and variance of the impulse response function of the APD are used, including their asymptotic behavior in time. Then the Gaussian-approximation 
approach enables us to capture the effect of ISI on the performance.

With this approximation, it is only necessary to calculate the mean and variance of $W$ under each hypothesis. Let $\mu_{0}$, $\sigma_{0}^{2}, \mu_{1}$, and $\sigma_{1}^{2}$ denote the mean and the variance under the hypothesis $H_{0}$ and $H_{1}$, respectively. Then the conditional pdf's are

$$
f_{i}(x)=\frac{1}{\sqrt{2 \pi} \sigma_{i}} \exp \left(-\left(x-\mu_{i}\right)^{2} / 2 \sigma_{i}^{2}\right), \quad i=0,1 .
$$

If $\theta_{1}$ and $\theta$ are the two distinct intersection points of the functions $f_{1}$ and $f_{0}$, with $\theta_{1}<\theta$, then the set $D$ defined in Section II-E is $\left(-\infty, \theta_{1}\right) \cup(\theta, \infty)$. Assuming $\sigma_{1}>\sigma_{0}$ and $\mu_{1}>\mu_{0}$, the integrals in (14) can be approximated by $\int_{D} f_{0}(x) d x \cong$ $\int_{\theta}^{\infty} f_{0}(x) d x$ and $\int_{D^{C}} f_{1}(x) d x \cong \int_{-\infty}^{\theta} f_{1}(x) d x$. (This approximation is valid since $\int_{-\infty}^{\theta_{1}} f_{0}(x) d x$ is negligible.) This is equivalent to replacing the optimal decision test in (13) by a threshold test for which (see (16a) and (16b) shown at the bottom of the page) and $\operatorname{erfc}(x)=1 / \sqrt{\pi} \int_{x}^{\infty} \exp \left[-y^{2}\right] d y$ is the complementary error function. Equations (10a) and (11a) along with the relations $E\left[W \mid H_{i}\right]=-j \varphi_{W}^{i}\left(0^{+}\right)$and $E\left[W^{2} \mid H_{i}\right]=-\varphi_{W}^{i^{\prime \prime}}\left(0^{+}\right)$are used to compute the true mean and the true variance under each hypothesis (the prime denotes the derivative and $\varphi\left(0^{+}\right)=\lim _{x \downarrow 0} \varphi(x)$.)

We now consider a special case of an integrate-and-dump circuit for which

$$
h(t)= \begin{cases}1, & 0 \leq t \leq T \\ 0, & \text { elsewhere }\end{cases}
$$

We further assume that the optical pulse is of rectangular shape of width $\tau$;

$$
p(t)= \begin{cases}\frac{n_{\circ}}{\tau}, & t \leq \tau \\ 0 & \text { elsewhere }\end{cases}
$$

where $n_{o}$ is the mean number of photon arrivals in the " 1 " bit. Taking $t_{S}=T$, the following expressions are obtained:

$$
\begin{aligned}
\mu_{0} & =\frac{1}{2} n_{o} \sum_{n=1}^{\infty} R_{n} \\
\mu_{1} & =\mu_{0}+R_{0} n_{o} \\
\sigma_{0}^{2} & =\sigma^{2}+\frac{1}{2} n_{o}^{2} \sum_{n=1}^{\infty}\left(R_{n}^{2}+C_{n}\right) \\
\sigma_{1}^{2} & =\sigma_{0}^{2}+n_{o} C_{0}
\end{aligned}
$$

where

$$
R_{n}=\int_{n T-\tau}^{n T} \bar{h}(s+T) d s
$$

and

$$
C_{n}=\int_{n T-\tau}^{n T} h_{2}(s+T) d s .
$$

The functions $\bar{h}$ and $h_{2}$ are given by

$$
\bar{h}(t)=\int_{t-T}^{t} E[I(s)] d s
$$

and

$$
h_{2}(t)=\int_{t-T}^{t} \int_{t-T}^{t} E\left[I\left(s_{1}\right) I\left(s_{2}\right)\right] d s_{1} d s_{2} .
$$

The mean impulse response function $i(t)=E[I(t)]$ for stable APD's has been shown [2] to have an exponentially decaying tail with rate $\gamma$. Thus, for large $t$ (in comparison to the sum of the electron and hole transit times in the APD denoted by $\left.\tau_{0}\right), i(t) \sim a e^{-\gamma t}$, where $a$ and $\gamma$ are known constants. For sufficiently large $n$ (i.e., $n \gg\left(\tau_{0}+\tau\right) / T$ ), one can use the above asymptotic result to evaluate $R_{n}$ :

$$
R_{n} \approx \frac{a}{\gamma^{2}}\left(1-e^{-\gamma T}\right)\left(e^{\gamma \tau}-1\right) e^{-\gamma n T}
$$

and hence for some sufficiently large integer $N$

$$
\sum_{n=1}^{\infty} R_{n} \approx \sum_{n=1}^{N} R_{n}+\frac{a}{\gamma^{2}}\left(e^{\gamma \tau}-1\right) e^{-\gamma N T}
$$

and

$$
\sum_{n=1}^{\infty} R_{n}^{2} \approx \sum_{n=1}^{N} R_{n}^{2}+\frac{a^{2}\left(e^{\gamma \tau}-1\right)^{2}\left(1-e^{-\gamma T}\right) e^{-2 \gamma N T}}{\gamma^{4}\left(1+e^{-\gamma T}\right)} .
$$

The situation is more complicated for $h_{2}$ due to the correlation function of the impulse response $E\left[I\left(t_{1}\right) I\left(t_{2}\right)\right]$ which appears in the expression for $h_{2}$. One can evaluate the correlation function numerically using the renewal relations that were derived in [20]. These expressions are rather long and we can use Schwarz inequality to find an upper bound in terms of the second moment of the impulse response. In this way, we can make use of our knowledge of the asymptotic behavior of the second moment that has been determined in [2]. Schwarz inequality gives the relation

$$
\begin{aligned}
E\left[I\left(t_{1}\right) I\left(t_{2}\right)\right] & \leq E\left[I^{2}\left(t_{1}\right)\right]^{1 / 2} E\left[I^{2}\left(t_{2}\right)\right]^{1 / 2} \\
& \triangleq \sqrt{i_{2}\left(t_{1}\right) i_{2}\left(t_{2}\right)} .
\end{aligned}
$$

But for large $t\left(t \gg \tau_{0}\right), i_{2}(t) \sim b e^{-\gamma t}$, for some known $b$. This decay rate is equal to that of the first moment. This is due to the fact that $I(t)$ depends on integer-valued random

$$
\mathrm{BER} \cong \frac{1}{2}\left\{\operatorname{erfc}\left(\frac{\theta-\mu_{0}}{\sqrt{2} \sigma_{0}}\right)+\operatorname{erfc}\left(\frac{\mu_{1}-\theta}{\sqrt{2} \sigma_{1}}\right)\right\}
$$

where

$$
\theta=\frac{-\frac{\mu_{1}}{\sigma_{1}^{2}}+\frac{\mu_{0}}{\sigma_{0}^{2}}+\sqrt{\left(\frac{\mu_{1}}{\sigma_{1}^{2}}-\frac{\mu_{0}}{\sigma_{0}^{2}}\right)^{2}-\left(\frac{1}{\sigma_{0}^{2}}-\frac{1}{\sigma_{1}^{2}}\right)\left\{\left(\frac{\mu_{0}^{2}}{\sigma_{0}^{2}}-\frac{\mu_{1}^{2}}{\sigma_{1}^{2}}\right)-2 \log \left(\frac{\sigma_{1}}{\sigma_{2}}\right)\right\}}}{\frac{1}{\sigma_{0}^{2}}-\frac{1}{\sigma_{1}^{2}}}
$$


processes, and hence the decay rate of its second moment must be at most equal to the decay rate of the first moment, in which case the relations characterizing the rate are identical for the first and second moments [2]. Thus, for some sufficiently large integer $N$,

$$
\sum_{n=1}^{\infty} C_{n} \approx \sum_{n=1}^{N} C_{n}+\frac{4 b^{2}\left(e^{(1 / 2) \gamma T}-1\right)^{2}\left(1-e^{-\gamma \tau}\right) e^{-\gamma N T}}{\gamma^{3}\left(1-e^{-\gamma T}\right)}
$$

Now the mean and variance under each hypothesis can be computed using (17), and the BER can be calculated from (16). Numerical results of the above analysis are presented in Section V.

\section{INSTANTANEOUS-RESPONSE APD}

Whereas the Gaussian approximation permitted us to include the time dynamics of the APD's response in the BER calculations, its accuracy is questionable at low optical power or high data rates. In this section, we develop an exact formulation but we ignore time dynamics. We assume an APD with an instantaneous response and use the probabilistic analysis of the gain of the APD including the effect of dead space [14].

This analysis assumes that APD's impulse response is sufficiently narrow to be considered as an impulse in comparison to the duration of each bit. The strength of this impulse is equal to the random gain of the APD multiplied by the electron charge $q$. In this case, the photodetector output $X$ is $q$ times the number of carrier-pairs generated as a result of the absorption of the photons in an optical pulse. Therefore, $X=\sum_{i=1}^{N} G_{i}$, where $\left\{G_{i}\right\}$ is an independent and identically distributed sequence of integer-valued random gains, and $N$ is the number of photons detected in a " 1 " bit, assumed to be Poisson with parameter $n_{o}$.

The characteristic function of the random variable $X$ is [16]

$$
\varphi_{X}(u)=\exp \left(n_{o}\left[\varphi_{G}(u)-1\right]\right)
$$

where $\varphi_{G}(u)=E[\exp (j u G)]$ is the characteristic function of $G$. The receiver output $W=X+V$ has a characteristic function

$$
\varphi_{W}(u)=\varphi_{X}(u) \varphi_{V}(u)
$$

where $V$ is an independent zero-mean Gaussian noise with variance $\sigma^{2}$. Once the characteristic function of $G$ is evaluated, the conditional characteristic functions of the observed output $W$ can be computed using (24) and (25). The probability density functions can then be evaluated using (12), and the optimum threshold can be determined from which the BER is computed using (16). We now proceed to consider the characteristic function of $G$ for the APD including the effect of dead space.

\section{A. Characteristic Function of the Gain of the APD}

The characteristic function of the gain for the conventional APD model was obtained by Personick [4] and Mazo et al. [5]. Without the dead space, the avalanche multiplication process is Markovian--since the age of a carrier at a certain point does not affect its ability to reproduce. This assumption simplifies the analysis greatly. For the dead-space model, on the other hand, a carrier's ability to reproduce (or ionize) depends on its age-the multiplication process is, therefore, non-Markovian. In [14], a pair of nonlinear coupled integral equations (renewal equations) were derived from which the generating function of the gain can be determined. For $|s| \leq 1$, these integral equations are

$$
\begin{array}{r}
F_{Z}(s, x)=s e^{-\alpha\left(w-x-d_{e}\right)}+\alpha \int_{x+d_{e}}^{w} F_{Z}^{2}(s, \xi) F_{Y}(s, \xi) \\
\cdot e^{-\alpha\left(\xi-x-d_{e}\right)} d \xi, \quad x \in\left[0, w-d_{e}\right]
\end{array}
$$

and

$$
\begin{array}{r}
F_{Y}(s, x)=s e^{-\beta\left(x-d_{h}\right)}+\beta \int_{0}^{x-d_{h}} F_{Y}^{2}(s, \xi) F_{Z}(s, \xi) \\
\cdot e^{-\beta\left(x-\xi-d_{h}\right)} d \xi, \quad x \in\left[d_{h}, w\right]
\end{array}
$$

where $w$ is the width of the multiplication region and $Z(x)$ (whose generating function is $F_{Z}(s, x)=E\left[s^{Z(x)}\right]$ ) denotes the sum of the total number of electrons and holes that are generated as a result of one parent electron at location $x$ in the multiplication region. Similarly, $Y(x)$ is the corresponding sum when a single hole at location $x$ initiates the multiplication process. The parameters $\alpha$ and $\beta$ are the ionization coefficients for electrons and holes, respectively, and $d_{e}$ and $d_{h}$ are their respective dead spaces. The gain $G=\frac{1}{2}(1+Z(0))$ so that

$$
F_{G}(s)=\sqrt{s} F_{Z}(\sqrt{s}, 0) .
$$

A detailed discussion of these equations can be found in [14].

In this paper, we solve the above equations analytically for the special (limiting) case when the hole-to-electron ionization ratio $k=\beta / \alpha=1$ and $d_{e}=d_{h}=d$. We obtain, for the first time, an explicit analytical expression for the characteristic function of the gain--we defer the derivation to the Appendix and give the final result:

$$
\varphi_{G}(u)=e^{r(d-w)+(1 / 2) j u}
$$

where $r$ satisfies the implicit relation

$$
r+\alpha e^{r(3 d-w)+j u}-\alpha=0 .
$$

To evaluate the characteristic function of the gain for an arbitrary $k$ (including the case $k=1$ ), we found it easiest to numerically solve the basic renewal equations (26) and (27) using Picard iteration. We give an outline of this procedure. For each $s$ on the unit circle: i) $F_{Z}(s, x)$ and $F_{Y}(s, x)$ are set to zero everywhere in the interval $[0, w]$, with the two exceptions $F_{Z}(s, w)=F_{Y}(s, 0)=s$; ii) equation (27) is discretized, using a suitable mesh size, and then used to generate estimates of $F_{Y}(s, x)$ in the interval $[0, w]$; iii) using this estimate of $F_{Y}(s, x)$ in the discrete version of (26), an estimate of $F_{Z}(s, x)$ is generated in the interval $[0, w] ;$ iv) an improved estimate of $F_{Y}(s, x)$ is obtained by substituting in the discrete version of (27) the previously calculated estimate of $F_{Z}(s, x)$; and v) steps iii and iv are repeated until convergence is achieved. The characteristic function $\varphi_{G}(u)$ is computed by the use of (28) with $s=e^{j u}$. 


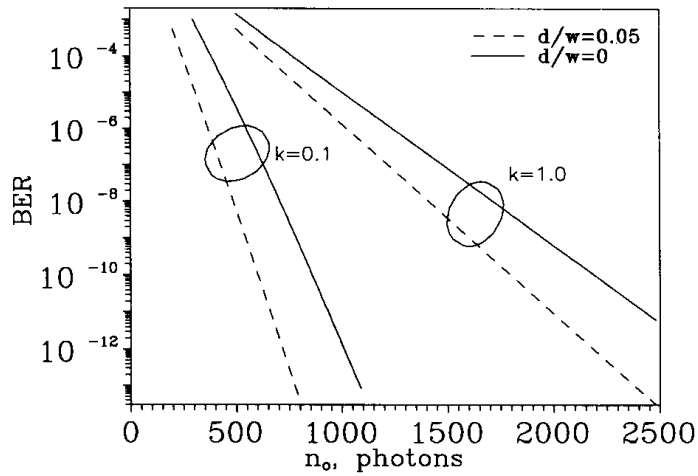

Fig. 2. The bit-error rate (BER) as a function of the mean number of photons per bit $n_{o}$ when a Gaussian approximation is used. The ratio of the dead space to the width of the multiplication region $d / w=0$ (solid) and $d / w=0.05$ (dashed). The APD's parameters are chosen so that the mean gain is 40 in all cases. Two cases are considered: an approximately single-carrier multiplication APD with $k=0.1$ and a relatively narrow impulse response (0.2 ns); and a double-carrier multiplication APD with $k=1.0$ and a wider impulse response $(0.4 \mathrm{~ns})$. The system is assumed to operate at $2 \mathrm{~Gb} / \mathrm{s}$ with a $50 \%$ duty cycle and the circuit-noise parameter $\sigma=500$.

\section{NumERICAL RESUltS AND DISCUSSION}

We compute the performance using the various approximations discussed in the previous sections. Two sets of results are obtained. In one we include the effects of the time response and dead space, but make the assumption that the receiver's photoelectric current is Gaussian. In the other approximation we assume that the detector response time is sufficiently short to be regarded as instantaneous, but use the exact statistics of the receiver output including the effect of dead space. In all the computations, the receiver filter $h$ is taken to have a rectangular impulse response function of width $T$ corresponding to an ideal integrator, and the optical pulse shape is also assumed to be rectangular of width $\tau$ and height $n_{o} / \tau$, the mean number of detected photons per bit (which is in turn proportional to the intensity of the light pulse [21]). When $\tau / T$ is small, photons arrive in a small cluster in each bit, so that the overlap among responses due to adjacent bits can be neglected, and therefore the instantaneousresponse approximation is valid. Whereas when $\tau / T$ is large, the Gaussian approximation is more suitable.

Figs. 2 and 3 depict the dependence of the BER on $n_{o}$ for the Gaussian and instantaneous-response approximations, respectively. The speed of the system is taken to be $2 \mathrm{~Gb} / \mathrm{s}$, i.e., $T=0.5 \mathrm{~ns}$, and the circuit noise parameter $\sigma=500$ (this value is within the typical range suggested in [22]). Two APD's of equal mean gains $(E[G]=40)$ are considered: an almost single-carrier multiplication APD $(k=0.1)$ with an impulseresponse width of $0.2 \mathrm{~ns}$, and a double-carrier multiplication APD $(k=1.0)$ with an impulse-response width of $0.4 \mathrm{~ns}$. The width of the impulse response function is taken to be the time necessary for the mean impulse response to build up $95 \%$ of the mean gain. Two sets of curves are generated corresponding to two values of the normalized dead space: $d / w=0$ and $d / w=0.05$. It is seen from these figures that dead space lowers each curve entirely so that the BER is reduced for each $n_{o}$.

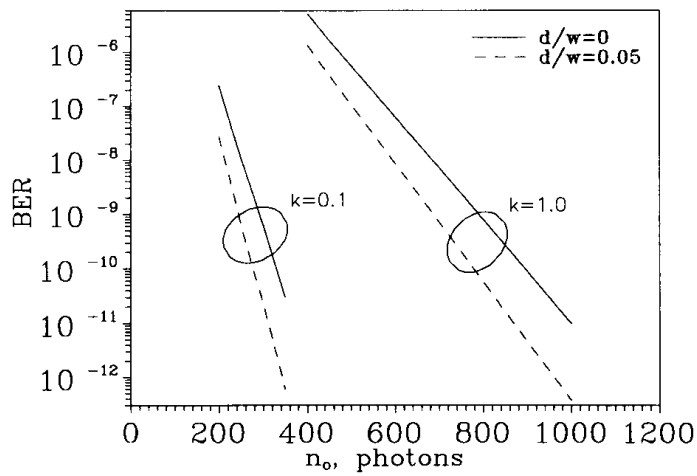

Fig. 3. The exact bit-error rate (BER) as a function of the mean number of photons per bit $n_{o}$ for an instantaneous receiver. The ratio of the dead space to the width of the multiplication region $d / w=0$ (solid) and $d / w=0.05$ (dashed). The APD's parameters are chosen so that the mean gain is 40 in all cases. Two cases are considered: an approximately single-carrier multiplication APD with $k=0.1$; and a double-carrier multiplication APD with $k=1.0$. The circuit-noise parameter $\sigma=500$.

It is customary to measure the performance of the receiver by its sensitivity $\left(n_{s}\right)$ which is typically defined to be the minimum mean number of photons per bit necessary to produce a BER of $10^{-9}$. The dependence of the sensitivity of the receiver on the channel dispersion can be examined by plotting the sensitivity as a function of the duty cycle of the transmitted signal $\tau / T$ as in Fig. 4. It is seen from Fig. 4 that with the Gaussian approximation, dead space enhances the performance for small values of $\tau / T$. As $\tau / T$ approaches unity, the sensitivity starts to increase (i.e., the performance becomes poorer) and dead space starts to degrade the performance. The former is simply the result of the orderliness, brought about by dead space, in the avalanche multiplication process. The latter, however, is due to the fact that dead space increases the duration of the impulse response function and hence increases in the overlap from adjacent bits. For small values of $\tau / T$, the sensitivity becomes constant since the interference from adjacent bits is negligible. In this regime, the Gaussian approximation is of no value since the exact analysis is available.

It is interesting to compare the receiver sensitivity of the device mentioned above to other idealistic devices mentioned in the literature. We have computed $n_{s}$ as a function of the data-transmission rate $B$ for a number of cases. The simplest case is that for which there is no gain noise (i.e., deterministic multiplier) but the response time is finite. This is shown in the lower curve denoted "finite response/deterministic gain" in Fig. 5. As $B$ increases, the sensitivity increases and the finite response of the APD becomes more effective in causing the deterioration of the performance. This curve provides a benchmark separating the effects of response time and gain fluctuations.

Another ideal device is that for which the response is instantaneous but the gain fluctuates. The results are shown by a set of three flat lines denoted "instantaneous response." The lower two lines have been computed using exact statistics of the gain. For comparison, the upper line was generated using a Gaussian approximation with the correct mean and variance. 


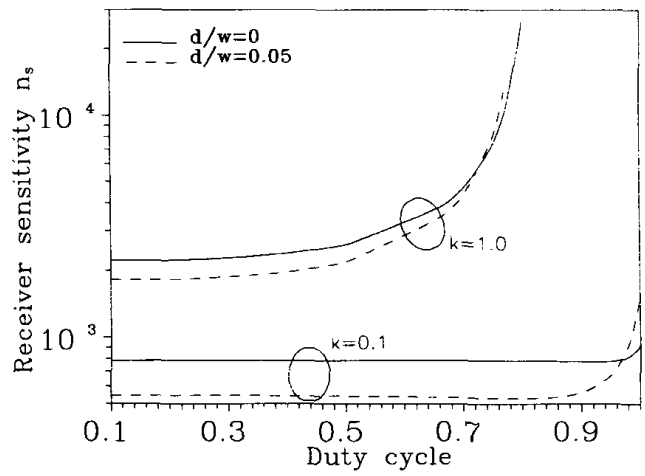

Fig. 4. The receiver sensitivity $n_{s}$ as a function of the duty cycle of the transmitted signal. The ratio of the dead space to the width of the multiplication region $d / w$ is taken to be 0 when no dead space is present (solid), and $d / w=0.05$ when dead space is present (dashed). The APD's parameters are chosen so that the mean gain is 40 . The data rate is assumed to be $2 \mathrm{~Gb} / \mathrm{s}$ and the circuit noise parameter $\sigma=500$. Two cases are considered for the hole-to-electron ionization ratio $k: k=0.1$; and $k=1.0$.

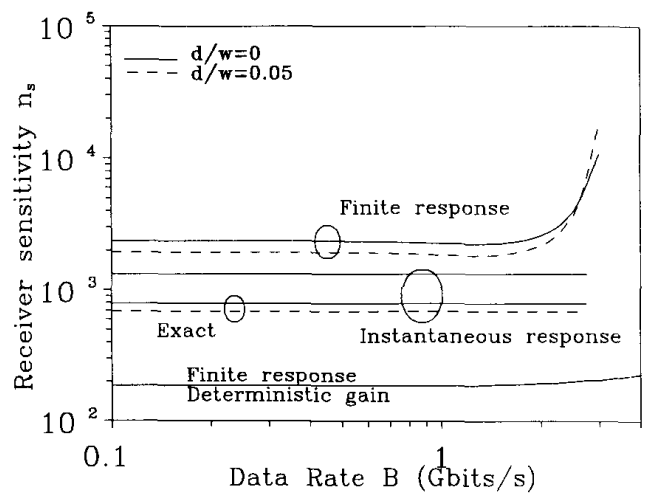

Fig. 5. Receiver sensitivity $n_{s}$ as a function of the transmission rate $B$ for a number of special cases. In the first case, for which the APD has deterministic gain with a deterministic finite response (equal to the mean impulse response function), the receiver has the best performance (lowest $n_{s}$ ). The second case (the set denoted instantaneous response), is that for which the APD is assumed to be instantaneous but with random gain. The lower two curves of this set are computed exactly, and the upper one is computed using the Gaussian approximation. Finally, the third set of curves, denoted finite response, represents a realistic device for which the gain is random and the response time is finite and random. The ratio of the dead space to the width of the multiplication region is $d / w=0$ (solid) and $d / w=0.5$ (dashed). In all cases, the mean gain of the APD is 40 , the ionization ratio $k=1$, the circuit-noise parameter $\sigma=500$, and the transmission duty cycle is $50 \%$.

It is clear that the Gaussian approximation underestimates the performance (or, equivalently, overestimates $n_{s}$ ). By comparing this set to the finite-response-deterministic-gain case, it is clear that gain fluctuations increase the sensitivity by about two orders of magnitude, whereas the increase in $n_{s}$ due to the finite response alone is less than one order of magnitude.

Finally, the third set of curves represents a realistic APD for which both the gain fluctuations and the finite response are included. By necessity, however, the Gaussian approximation is used and the results are more accurate for large values of $n_{o}$.

In all of the computations of interest, we have included a comparison between devices with and without dead space, but with the same mean gain. It is clear that under this constraint, the dead-space device is superior at most by a factor of 1.3 for relatively low $B$ (i.e., for $T$ greater than the width of the impulse response). This is a result of inhibition and orderliness effects due to dead space which reduces variation noise in the multiplication process. A similar enhancement in the signalto-noise ratio was also reported earlier [14] and [15]. Once again, the negative effect of dead space on the performance is seen for very high data rates. As before, this is due to the longer tails of the impulse response function which results in a longer response time.

\section{CONCLUSION}

Two effects complicate the study of bit-error rates in optical receivers using APD's-dead space of the avalanching process, and the finite and random response time of the device. We have determined the performance of such receivers in an on-off keying optical communication system including for the first time these effects. We found that the presence of dead space corresponds to lower bit-error rates for relatively low data rates when the mean gain of the APD is kept constant. Dead space, therefore, enhances the performance at these rates due to the reduction in the APD gain-fluctuation noise as a result of the inhibition and orderliness brought about by dead space. On the other hand, for very high data rates, dead space degrades the performance since it is responsible for longer tails in the impulse response function of the APD. In this case, dead space contributes to intersymbol interference.

It was also found that at high data rates, the dominant factor that controls the receiver performance is the finite response time of the receiver, while for low data rates, the performance is governed by the fluctuations in the APD gain.

For computations in the high-data regime, we employed a Gaussian approximation with exact mean and standard deviation of the receiver output. For low data rates, the exact statistics of the receiver output were computed and used to determine the performance. This was only possible under the assumption that the receiver was instantaneous.

It is of interest, therefore, to design devices for which dead space is enhanced when the data rate is low and reduced when operating at a high rate. New technologies using multilayer structures may possibly be employed to achieve this goal.

\section{APPENDIX}

We start by differentiating (26) and (27) with respect to $x$ to obtain the following differential equations:

$$
\begin{aligned}
\partial F_{Z}(s, x) / \partial x= & \alpha\left(F_{Z}(s, x)-F_{Z}^{2}\left(s, x+d_{e}\right)\right. \\
\cdot & \left.F_{Y}\left(s, x+d_{e}\right)\right), \quad x \in\left[0, w-d_{e}\right] \\
\partial F_{Y}(s, x) / \partial x=- & \beta\left(F_{Y}(s, x)-F_{Y}^{2}\left(s, x-d_{h}\right)\right. \\
& \left.\cdot F_{Z}\left(s, x-d_{h}\right)\right), \quad x \in\left[d_{h}, w\right]
\end{aligned}
$$

with the following boundary conditions:

$$
F_{Z}\left(s, w-d_{e}\right)=s,
$$

and

$$
F_{Y}\left(s, d_{h}\right)=s .
$$

Under the assumption that $k=1$ (i.e., $\alpha=\beta$ ) and $d_{e}=d_{h}=$ $d$, one can easily verify from (A.1)-(A.3) that $F_{Y}(s, x)=$ 
$F_{Z}(s, w-x)$. Hence, from (A.1), we obtain

$$
\begin{aligned}
\alpha^{-1} \partial F_{Z}(s, x) / \partial x=F_{Z}(s, x)- & F_{Z}^{2}(s, x+d) \\
& \cdot F_{Z}(s, w-x-d) .
\end{aligned}
$$

Assume a solution of the form

$$
F_{Z}(s, x)=\hat{c} e^{r_{s} x}
$$

and substitute (A.5) into (A.4) to obtain the characteristic equation

$$
r_{s}+\alpha \hat{c} e^{r_{s}(w+d)}-\alpha=0 .
$$

By applying the boundary condition (A.3a) to (A.5), we determine the constant $\hat{c}$ and obtain

$$
F_{Z}(s, x)=s e^{r_{s}(x+d-w)}
$$

where $r_{s}$ is the solution to the equation

$$
r_{s}+\alpha s^{2} e^{r_{s}(3 d-w)}-\alpha=0 .
$$

Since $G=\frac{1}{2}(1+Z(0))$,

$$
F_{G}(s)=\sqrt{s} F_{Z}(\sqrt{s}, 0)
$$

and hence we obtain the expression for the generating function of $G$ :

$$
F_{G}(s)=\sqrt{s} e^{(d-w)} r_{\sqrt{s}}
$$

Since the characteristic function of $G$ is simply $\varphi_{G}(u)=$ $F_{G}\left(e^{j u}\right)$, the results in (29) and (30) are therefore established.

\section{REFERENCES}

[1] S. D. Personick, Fiber Optics. New York: Plenum, 1985.

[2] M. M. Hayat and B. E. A. Saleh, "Statistical properties of the impulse response function of double-carrier multiplication avalanche photodiodes including the effect of dead space," J. Lightwave Technol., vol. 10, pp. 1415-1425, 1992.

[3] P. P. Webb and R. J. McIntyre, "Recent developments in silicon avalanche photodiodes," RCA Eng., vol. 27, pp. 96-102, 1982.

[4] S. D. Personick, "Statistics of a general class of avalanche detectors with application to optical communication," Bell Syst. Tech. J., vol. 50, pp. 3075-3095, 1971.

[5] J. E. Mazo and J. Salz, "On optical communication via direct detection of light pulses," Bell Syst. Tech. J., vol. 55, pp. 347-369, 1976.

[6] E. N. Gilbert and H. O. Pollak, "Amplitude distribution of shot noise," Bell Syst. Tech. J., vol. 39, pp. 333-350, 1960.

[7] C. W. Helstrom, "Computing the performance of optical receivers with avalanche detectors," IEEE Trans. Commun., vol. 36, pp. 61-66, 1988

[8] G. L. Cariolaro, "Error probability in digital fiber optic communication systems," IEEE Trans. Inform. Theory, vol. IT-24, pp. 213-221, 1978.

[9] M. Mansuripur, J. W. Goodman, E. G. Rawson, and R. E. Norton, "Fiber optic receiver error rate prediction using the Gram-Charlier series," IEEE Trans. Commun., vol. COM-28, pp. 402-407, Mar. 1980.

[10] S. D. Personick, P. Balaban, and J. H. Bobsin, "A detailed comparison of four approaches to the calculation of the sensitivity of optical fiber system receivers," IEEE Trans. Commun., vol. COM-25, pp. 541-548, 1977.

[11] M. M. Hayat, "Bit-error-rates for optical receivers using avalanche photodiodes," Masters thesis, Univ. Wisconsin-Madison, 1988.

[12] K. F. Brennan, Y. Wang, M. C. Teich, B. E. A. Saleh, and T. Khorsandi, "Theory of the temporal response of a simple multiquantumwell avalanche photodiode," IEEE Trans. Electron. Devices, vol. ED-35, pp. 1456-1467, 1988 .

[13] K. B. Letaief and J. S. Sadowsky, "Computing bit-error probabilities for avalanche photodiode receivers by large deviation theory," IEEE Trans. Inform. Theory, vol. 38, pp. 1162-1169, 1992.
[14] M. M. Hayat, B. E. A. Saleh, and M. C. Teich, "Effect of dead space on gain and noise of double-carrier-multiplication avalanche photodiodes," IEEE Trans. Electron Devices, vol. 39, pp. 546-552, 1992.

[15] M. M. Hayat, W. L. Sargeant, and B. E. A. Saleh, "Effect of dead space on gain and noise in $\mathrm{Si}$ and GaAs avalanche photodiodes," IEEE $J$. Quantum Electron., vol. 38, pp. 1360-1365, 1992.

[16] D. L. Snyder, Random Point Processes. New York: Wiley, 1975

17] J. A. Gubner, "Computation of shot-noise probability distributions and densities," SIAM J. SCi. Comput., to appear.

[18] A. Papoulis, "High density shot noise and Gaussianity," J. Appl. Prob., vol. 8 , pp. $118-127,1971$.

[19] S. D. Personick, "Receiver design for optical fiber systems," Proc. IEEE, vol. 65 , pp. 1670-1678, 1977.

[20] M. M. Hayat, "Performance of optical communication systems using avalanche photodiodes with dead space," Ph.D. thesis, Univ. WisconsinMadison, 1992.

[21] B. E. A. Saleh and M. C. Teich, Fundamentals of Photonics. New York: Wiley, 1991.

[22] S. D. Personick, "New results on avalanche multiplication statistics with applications to optical detection," Bell Syst. Tech. J., vol. 50, pp. 167-189, 1971.

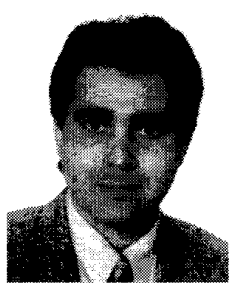

Majeed M. Hayat (S'88-M'92) was born in Kuwait in 1963. He received the B.S. degree (summa cum laude) in electrical engineering from the University of the Pacific, Stockton, CA, in 1985, and the M.S. and Ph.D. degrees in electrical and computer engineering in 1988 and 1992, respectively, from the University of Wisconsin-Madison.

He is currently a Postdoctoral Research Associate in the Department of Electrical and Computer Engineering at the University of Wisconsin-Madison. His research interests include statistical communication theory, performance analysis of optical communication systems, point processes, and quantum noise in optical networks.

Dr. Hayat is a member Eta Kappa Nu, Phi Kappa Phi, and Tau Beta Sigma.

Bahaa E. A. Saleh (M'73-SM'86-F'91) received the B.S. degree from Cairo University in 1966, and the Ph.D. degree from the Johns Hopkins University, Baltimore, MD, in 1971, both in electrical engineering.

From 1971 to 1974 he was an Assistant Professor at the University of Santa Catarina, Brazil, and from 1974 to 1976 he was a Research Associate at the Max Planck Institute, Göttenberg, Germany. Since 1977 he has been on the faculty of the Department of Electrical and Computer Engineering at the University of Wisconsin-Madison, and he has served as department chairman from 1990 to 1994 . He is currently professor and chairman of the Electrical, Computer and Systems Engineering Department at Boston University. He held visiting appointments at the University of California, Berkeley, and Columbia University. His research activities cover a broad spectrum of topics in optics and optoelectronics (statistical and quantum optics, optical signal processing, optical communication, nonlinear optics, photodetectors), digital image processing (enhancement and restoration), and vision (adaption). He is the author of two books, Photoelectron Statistics (Springer-Verlag, 1978), and Fundamentals of Photonics (Wiley-Interscience, 1991, with M. C. Teich), the Co-Editor of Transformation in Optical Signal Processing (SPIE, 1983), and the author of chapters in seven books. He is author or co-author of more than 150 papers in technical journals.

Dr. Saleh is the Editor-in-Chief of the Journal of the Optical Society of America A, member of the board of editors of the Journal of the European Optical Society B: Quantum Optics, and co-editor of the Adam Hilger Optics and Optoelectronics Series, Institute of Physics, UK. He is a Fellow of the Optical Society of America. He is also a member of Phi Beta Kappa and Sigma XI. In 1981 he received the University of Wisconsin Romnes Award, and in 1984 he became Fellow of the Guggenheim Foundation.

John A. Gubner (S'83-M'88) received the B.S., M.S., and Ph.D. degrees in electrical engineering from the University of Maryland, College Park, in 1983,1985 , and 1988, respectively.

In 1988 he joined the faculty of the University of Wisconsin-Madison, as an Assistant Professor in the Department of Electrical and Computer Engineering. In 1985, he received the 1EEE Frank A. Cowan Scholarship for graduate study in communications, and from 1986 to 1988 , he was a Graduate Fellow with the Systems Research Center at the University of Maryland. His research interests include information theory, shot-noise random processes, distributed estimation, and wavelets. 